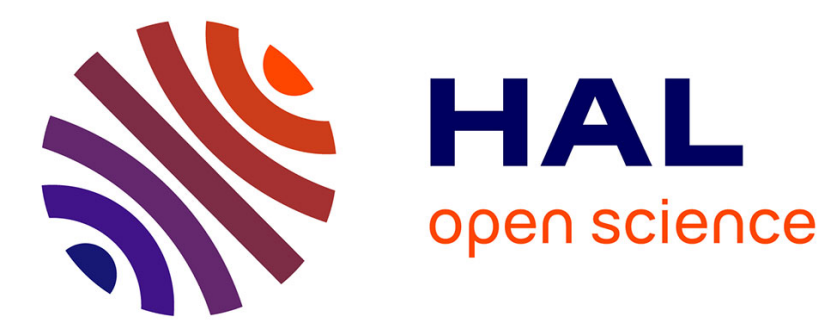

\title{
Nanoscale structural mapping as a measure of maturation in the murine frontal cortex
}

Georgiy Smolyakov, Etienne Dague, Clément Roux, M. H Seguelas, C. Galés, J. M. Senard, D. N. Arvanitis

\section{- To cite this version:}

Georgiy Smolyakov, Etienne Dague, Clément Roux, M. H Seguelas, C. Galés, et al.. Nanoscale structural mapping as a measure of maturation in the murine frontal cortex. Brain Structure and Function, 2018, 223 (1), pp.255-265. 10.1007/s00429-017-1486-z . hal-01907682

HAL Id: hal-01907682

https://hal.laas.fr/hal-01907682

Submitted on 22 Nov 2018

HAL is a multi-disciplinary open access archive for the deposit and dissemination of scientific research documents, whether they are published or not. The documents may come from teaching and research institutions in France or abroad, or from public or private research centers.
L'archive ouverte pluridisciplinaire HAL, est destinée au dépôt et à la diffusion de documents scientifiques de niveau recherche, publiés ou non, émanant des établissements d'enseignement et de recherche français ou étrangers, des laboratoires publics ou privés. 


\title{
Nanoscale structural mapping as a measure of maturation in the murine frontal cortex
}

Smolyakov $G^{\mathrm{a}, \mathrm{b} *}$., Dague $\mathrm{E}^{\mathrm{a}, \mathrm{b}^{* \mathfrak{x}}}$., Roux $\mathrm{C}^{\mathrm{c}}$., Seguelas $\mathrm{MH}^{\mathrm{d}}$., Gales $\mathrm{C}^{\mathrm{b}, \mathrm{d}}$., Senard JM${ }^{\mathrm{d}}$., Arvantis DN. ${ }^{\mathrm{d}^{*} \mathfrak{f}}$

\section{Authors affiliations :}

${ }^{a}$ LAAS-CNRS, Université de Toulouse, CNRS, Toulouse, France

${ }^{\mathrm{b}}$ ITAV-CNRS, Université de Toulouse, CNRS, Toulouse, France

${ }^{\mathrm{c}}$ Laboratoire des IMRCP, Université de Toulouse, CNRS UMR 5623, Université Paul Sabatier, 118 route de Narbonne 31062 Toulouse Cedex 9, France

${ }^{\mathrm{d}}$ Institut des Maladies Métaboliques et Cardiovasculaires, INSERM UMR1048, Université Paul Sabatier, 118 route de Narbonne 31062 Toulouse Cedex 9, France

* equally contributed

${ }^{£}$ corresponding authors

To whom correspondence should be addressed: constandina.arvanitis@univ-tlse3.fr and edague@laas.fr

Running title: Nanoscale structural mapping of the murine frontal cortex

Keywords: Frontal cortex, Atomic force microscopy, Perineuronal nets, Maturation

ORCHID: Arvantis D:0000-0003-4676-4455: Dague E: 0000-0003-3290-9166: Roux C: 0000-00015707-4471.

\begin{abstract}
$\underline{\text { Abstract }}$
The application of Atomic Force Microscopy (AFM) force mapping is emerging as an innovative means to phenotype the brain. This study shows the utility of AFM to determine nanomechanical and nanostructural features of the murine dorsolateral frontal cortex from weaning to adulthood. We show an increase in tissue stiffness of the primary somatosensory cortex with age, along with an increased cortical mechanical heterogeneity. To characterize the features that potentially produce this heterogeneity, we applied AFM scan mode to directly image the topography of thin sections of the primary somatosensory cortical layers II/III; IV and V/VI. Topographical mapping of the cortical layers at successive ages showed progressive smoothing of the surface. Topographical images were also compared with histochemically derived morphological information, which demonstrated the deposition of perineuronal nets, important extracellular components and markers of maturity. Our work demonstrates that high-resolution AFM images can be used to determine the nanostructural properties of cortical maturation, well beyond embryonic and postnatal development. Furthermore, it may offer a new method for brain phenotyping and screening to uncover topographical changes in early stages of neurodegenerative diseases.
\end{abstract}




\section{$\underline{\text { Introduction }}$}

Neuroimaging techniques of human and rodent brains have established that the functional and structural maturation of the frontal cerebral cortex (FC) transpires after adolescence: at approximately 20 years of age in humans and around postnatal day 50 in rodents (Marsh et al., 2008; Semple et al., 2013). These changes parallel improvements in both working memory and cognitive control. The key process known to govern this maturation is synaptic pruning, which leads to a reduction in cortical volume as well as an increase in myelination and tightening of the myelin sheaths (reviewed in (Croteau-Chonka et al., 2016; Giedd et al., 1999; Paus et al., 2008). Various studies have helped to uncover several of the complex mechanisms underlying the maturation process some of which include the role in brain activity, and the timing of shifts from immature to adult neuro-circuitry (Johnson et al., 2016a; Somerville, 2016). More recently, a comprehensive mass spectrometry based analyses of the lipid composition of prefrontal cortices in primates showed changes in over $60 \%$ of the lipidome occur during postnatal maturation prior to adulthood, with less than a quarter associated with myelination progression ( $\mathrm{Li}$ et al., 2017). Even with these findings, few structural markers exist to measure maturation.

One important line of research is the acquisition of inhibitory interneuron markers with the eventual deposition of the extracellular perineuronal nets (PNNs). PNNs are macromolecular structures predominantly composed of proteoglycans, hyaluronans, link proteins and tenascins (Zimmermann and Dours-Zimmermann, 2008). PNN deposition occurs during what are known as critical periods (Takesian and Hensch, 2013). Critical periods are transitional stages in which neural networks are particularly receptive, therefore more plastic, and can be shaped based on environmental influences or stimulation (Hensch, 2005). The deposition of PNNs mark the closure of the critical period, and have become the predominant indicators of maturation in the visual cortex (Bernard and Prochiantz, 2016; Di Cristo et al., 2007). Evidence for their refinement in the FC has also been shown. For instance, PNN deposition occurs in response to environmental conditions such as enrichment (Carstens et al., 2016), stress (Callaghan et al., 2013) or learning (Johnson et al., 2016b). In spite of these findings it is established that maturation of the juvenile PNN matrix may not be the result of the 
deposition of PNN components, as many of the PNN components are already present in the immature CNS before the formation and imaging of the network lattice (Carulli et al., 2007; Galtrey et al., 2008). As such, methods allowing for the micro- or nanostructural phenotyping of cortical maturation process remain limited.

Application of the AFM force mapping to neurobiology is unveiling a modern means for nanomechanical phenotyping of the brain. Aside from understanding the mechanical forces on individualized neurons (Franze et al., 2013; Spedden and Staii, 2013; Ungureanu et al., 2016), application of the AFM to neuroscience have demonstrated mechanical heterogeneity in numerous neuronal tissues from rodent, canine and human studies (Litinetsky et al., 2002; Zhang et al., 2013). Systemic biomechanical profiling of mouse tissue and cellular stiffness in the developing embryonic cortex showed a shift toward an increase in stiffness at successive developmental stages that correlates with the emergence of neurons and the establishment of cortical layers (Iwashita et al., 2014). Similarly, biomechanical profiling of rat cerebral cortices at early postnatal periods and in the adult has demonstrated a continued evolution of and shift toward stiffness in cerebral tissues (Elkin et al., 2010). Furthermore, the understanding of the mechanical properties of the cerebral cortex complement the plethora of cellular and molecular data to form a more complete picture of how the mechanical properties of brain tissue change with age, injury and disease (Elkin et al., 2007; Magdesian et al., 2012; Park et al., 2015; Wu et al., 2016; Yersin et al., 2007). Very recently it was shown that scarring of the rat cerebral cortex and spinal cord led to a soft mechanical signature following stab and crush injury; a property that appears unique to the CNS (Moeendarbary et al., 2017).

Here, we aimed to extend these measurements to determine whether the process of maturation of the neocortical primary somatosensory cortex (PSSC), a region with impressive postnatal maturation and intense staining with PNNS (Brückner et al., 1994; Fox and Wong, 2005), can be evaluated by Young's modulus. Young's modulus refers to the tensile modulus of a material, which is a measure of the stiffness and often used to characterize materials (reviewed in J. Roa et al., 2011). Using fresh or mildly fixed slices of brain, which rendered a detailed evaluation of the frontal cortex related to the PSSC layers II/III; IV and V/VI, we observed successive increase in stiffness together with an 
increase in the heterogeneity over a period of postnatal maturation from postnatal day 20 (P20), through juvenile stage day 45(P45) to the adult cortex, day 90 (P90). Next, we sought to apply the AFM contact mode to visualize the surface topography of PSSC slices to determine whether such images can provide crucial information on the maturation processes. Contact mode topographical screening is emerging as a gold standard technique to collect more detailed information about surface and interface properties respect to other microscopy techniques (Burnham and Colton, 1998; Marrese et al., 2017). Application of the contact mode to cortical slices conventionally used for histology allowed for nanoscale topographical analyses of the PSSC. We show that contact mode phenotyping of the nanostructure of neuronal tissues allowed us to observe detailed changes in the tissue architecture that correlate to numerous changes one of which is the deposition of the PNNs.

\section{$\underline{\text { Experimental Procedures }}$}

Animals. Experimental animals were housed with food and water available ad libitum under standard $12 \mathrm{~h}$ light/dark cycles. Animals used were inbred C57B6J wild-type males at three developmental ages ranging from weaning at postnatal days 17-20 (P20), juvenile at postnatal 42-45 days (P45) and adult at 85-90 days old (P90).

Brain Tissue Preparation. Briefly, mice were anesthetized with isofluorane, decapitated and the FC (brain sections corresponding to stereotaxic location: interaural $4.90 \mathrm{~mm}$, bregma $1.10 \mathrm{~mm}$ to interaural 4.18mm, bregma $0.38 \mathrm{~mm}$ : see flow chart in Figure 1A) were sectioned in ice-cold PBS. For topography studies, the tissue was placed in $4 \%$ PFA for $24 \mathrm{~h}$, then paraffin embedded and serial cuts of $5 \mu \mathrm{m}$ coronal sections were obtained. Prior to topographical mapping studies, typical rehydration process for histochemical analyses was performed. Briefly, slides were deparaffinized with xylene, rehydrated in decreasing concentrations of ethanol, placed in ice-cold PBS and topography was analyzed within $2 \mathrm{~h}$.

For force mapping studies, brain tissues from each age group was removed, and $400 \mu \mathrm{m}$ coronal sections (brain sections corresponding to stereotaxic location: interaural $4.90 \mathrm{~mm}$, bregma $1.10 \mathrm{~mm}$ to interaural $4.18 \mathrm{~mm}$, bregma $0.38 \mathrm{~mm}$ ) were obtained using a vibratome (752 Vibroslice Tissue Cutter by Campden Instruments Ltd, UK.). Fresh brain sampling was performed as previously described 
(Nieto-Gonzalez and Jensen, 2013), the brain was removed rapidly brains were dissected out and transferred to ice-cold artificial cerebrospinal fluid (aCSF) oxygenated and composed of $126 \mathrm{mM}$ $\mathrm{NaCl}, 2.5 \mathrm{mM} \mathrm{KCl}, 2 \mathrm{mM} \mathrm{CaCl}_{2}, 2 \mathrm{mM} \mathrm{MgCl}_{2}, 1.25 \mathrm{mM} \mathrm{NaH}_{2} \mathrm{PO}_{4}, 26 \mathrm{mM} \mathrm{NaHCO}_{3}, 10 \mathrm{mM}$ D-glucose at $\mathrm{pH}$ 7.4. Slices were stored in the aCSF solution supplemented with $3 \mathrm{mM}$ kynurenic acid for $1 \mathrm{~h}$ and processed by AFM within $2 \mathrm{~h}$. For mildly fixed samples the slices were incubated in artificial cerebral spinal fluid (aCSF; (MacGregor et al., 2001)) for 1 hour and fixed in 1\% PFA for 1 hour, rinsed and stored on ice in PBS and processed within $4 \mathrm{~h}$.

The sections were glued (SADER Resin) onto tissue culture plates (TPP, Switzerland) and covered with supplemented aCSF for live tissues or cooled PBS for mildly fixed tissues. Right and left cortical hemispheres were arbitrarily studied for each age. Cortical distances were first determined by measuring optically the distance from the edge of the cortex to white matter tracts at the corpus callosum. For fresh tissues, 45-50 spots were arbitrarily mapped with in the entire cortical region. For mildly fixed tissues, the distance between the edge of the cortex to white matter tracts was divided into three, resulting in approximately readings at each $300 \mu \mathrm{m}$ from the outer contour of the cortex. Three readings were obtained for each cortical area per slice, and 2 cortical slices at indicated frontal areas were studied per animal (n=3/group for fresh slices and n=4/group for mildly fixed slices).

Histochemistry. For histochemical analyses selected sections were rinsed for $30 \mathrm{~min}$ in $0.1 \mathrm{M}$ PBS followed by a blocking solution (DAKO Serum free protein block) for $1 \mathrm{~h}$ at room temperature. Sections were incubated for $1 \mathrm{~h}$ with Wisteria Floribunda Lectin FITC conjugated (WFA, 1:500; Thermofischer) in 0.1M PBS-0.05\% TritonX-100 (PBST) and nuclei were counterstained with Dapi (1:1000, Sigma). Sections were rinsed with PBST for 30 min then PBS for 30min. The samples were stored in PBS, visualized for WFA staining using a Jenoptik ProgRes® M camera and processed for height image analyses and then dried and cover-slipped with mounting media (DAKO). Sections were further viewed and images were captured on a Digital Slice Scanner Nanozoomer (Hamamatsu). Acquisition of images was performed using the NDP viewer software.

Fabrication of microsphere-modified cantilevers, AFM height and mechanical measurements 
Silica microspheres of $5 \mu \mathrm{m}$ diameter (PSI-5.0,Kisker Biotech) were glued by adhesive photopolymer NOA63 from Norland Products to bare NP-O10-D cantilevers (Bruker) as previously described (Beaussart et al., 2014). Briefly, cantilevers are coated with a photopolymer glue before contacting a microsphere. Once the microsphere is on the cantilever, the glue is polymerized under UV irradiation and the colloidal probe is ready for force measurements on brain slices.

A Nanowizard III from JPK AFM was used for the present study. Height images were obtained in contact mode by using the following scanning conditions: force set point $5 \mathrm{nN}$, scanning frequency $1 \mathrm{~Hz}$, image resolution 512 pixels on a scan area of $100^{2} \mu \mathrm{m}^{2}$. The mechanical measurements, were done in force spectroscopy mode with the following settings: force set point $3 \mathrm{nN}$, ramp size $10 \mu \mathrm{m}$, tip velocity $20 \mu \mathrm{m} / \mathrm{s}$. Approximately 40 spots throughout the entire PSSC or 30 spots in $100^{2} \mu \mathrm{m}^{2}$ size zones related to the layers of the PSSC were indented in each case, with 5 force curves per spot being collected. MLCT-C cantilevers with a nominal spring constant of $10 \mathrm{mN} / \mathrm{m}$ were used for imaging, while the microsphere-modified NP-O10-D cantilevers with a nominal spring constant of $60 \mathrm{mN} / \mathrm{m}$ were applied for mechanical measurements. The thermal tune method (Emerson and Camesano, 2006) was used to calibrate the spring constant of a particular cantilever before each experiment. The JPK Data Processing software was used for the data analysis. The subtraction of polynomial fit of $1^{\text {st }}$ order from each scan line was done for the height images. In turn, the force curves were subject to the base line adjustment before calculating tip-surface separation and further elastic modulus. Hertz elastic model for spherical contact between tip and surface (Hertz, 1882) was used for the analysis of the force curves. This model links the sample deformation $\delta$ to the applied force $F$ via elastic modulus $E$ and tip (microsphere) radius $R$ values:

$$
\begin{aligned}
& F=\frac{4}{3} \cdot \frac{E}{1-v^{2}} \cdot R^{\frac{1}{2}} \cdot \delta^{\frac{3}{2}} \text { (equation 1) } \\
& F=4 / 3 * E /\left(1-v^{2}\right) * R^{1 / 2} * d^{3 / 2} \text {, with } v \text { being sample Poisson ratio, arbitrary taken as } 0.5 .
\end{aligned}
$$

Surface root mean squared roughness $(\mathrm{Rq})$ were evaluated using the JPK data processing software

$$
R q=\sqrt{\frac{1}{n} \sum_{i=1}^{n} y_{i}^{2}}(\text { equation 2) }
$$


Statistical Analyses. For force curves, all data points were plotted and the median for each age and layer were determined. Significant differences are shown as evaluated by 2 -way ANOVA $* \mathrm{P}<0.05$, $* * \mathrm{P}<0.01$ and $* * * \mathrm{P}<0.001$. For $\mathrm{Rq}$ analyses, 2 -way ANOVA was performed. In an effort to limit experimenter bias in the analysis of topographical features of the cortex slices, we implemented a simple image analysis strategy using ImageJ. Contact-mode images were exported to tiff format and converted and downscaled to 8-bit. When necessary, a simple plane-fit was applied. Images were then binarized at a constant threshold and analysed using the particle analysis routine in ImageJ. The range of areas considered was $0-6 \mu \mathrm{m}^{2}$, which automatically discarded most soma from the analyses. The circularity factor was set at the 0.2-1 range. 3 images were analysed for each condition.

\section{RESULTS}

Biomechanical heterogeneity of frontal dorsolateral cortices increases with age:

To identify if the maturation of the primary somatosensory cortex can be characterised by an age-related shift in tissue stiffness, $400 \mu \mathrm{m}$ sections corresponding to the indicated stereotaxic areas were isolated from late postnatal (P20), juvenile (P45) and adult (P90) mice, and analysed by AFM for Young's modulus calculation. An AFM colloidal probe was used and measurements were obtained by 40-50 dispersed points throughout the PSSC. A schematic representation of the methodology is shown in figure 1A. Young's moduli corresponding to each point was then calculated from the obtained forcedistance curves and plotted in Figure 1B. An AFM colloidal probe was used and measurements were obtained by approximately 45 dispersed points throughout the entire PSSC. A schematic representation of the methodology is shown in Figure 1A. Young's moduli corresponding to each point was then calculated from the obtained force-distance curves and plotted in Figure 1B for fresh tissues and Figure $1 \mathrm{C}$ for mildly fixed tissues.

We calculated, from the force versus indentation curves, the elastic moduli for each age and found a median modulus of $209 \pm 15$ PA for the PSSC of weaned animals, a median modulus of $509 \pm 53$ PA for the PSSC isolated from juveniles and a mean modulus of 790 77 PA for adult cortices (Figure 1B), which are consistent with previously published findings showing an age-dependant increase in the 
stiffness of the PSSC. In addition to the increase in stiffness, we noted heterogeneity in the stiffness values per age. One difficulty encountered with fresh tissues was the surface contamination of the probe that limited a systemic and more thorough examination of the mechanical properties of the cortical area. To overcome this and identify if the maturation of the primary somatosensory cortex can be characterised by an age-related shift in tissue stiffness in specific cortical layers, we performed similar experiments on mildly fixed tissues. For these experiments, we used an AFM colloidal probe and 35-40 dispersed points throughout $100^{2} \mu \mathrm{m}^{2}$ area within layer II/III, IV and V/VI were obtained allowing for more comprehensive analyses of the cortical regions. For mildly fixed slices we calculated, from the force versus indentation curves, the elastic moduli for each age and found a median modulus of $245 \pm 54$ PA for the PSSC of weaned animals, a median modulus of $750 \pm 97$ PA for the PSSC isolated from juveniles and a mean modulus of 1150 \pm 161 PA for adult cortices (Figure 1C). These values are consistent with those previously published for cortex (Elkin et al., 2010). We further note a significant increase in the modulus measured from P20 to P90 indicating roughly a 4.5-fold increase in stiffness in the FC from weaning to adult stages (Figure 1C). Of note, in both fresh and mildly fixed slides we observed that the changes in stiffness were not due to the removal of elastic material, as force curves ranging from 55 PA to 250 PA were observed within all groups. Rather, the scatter plots indicate that with each successive age there is the addition of stiffer elements, which results in the increase of the cortical mechanical heterogeneity (Figure 1B and 1C).

\section{$\underline{\text { Contact mode analyses of fine brain sections: }}$}

An increase in the deposition of PNNs is known to occur from weaning to adulthood in several brain regions. Histochemical analysis was performed using Fluorescein Wisteria Floribunda Lectin (WFA, Vector Laboratories) to stain PNNs and DAPI as a nuclear counterstain. Figure 2 (A, B and C) confirm the age-dependant deposition of PNNs in the PSSC. Figure D to F are magnified images (white square on Figure 2A to C) of the layer IV dorsolateral cortices: a region where we showed an increase in stiffness and heterogeneity with age. We observed the deposition of PNNs occurs after P20 (Figure 2A and D) whereas diffuse staining is observed at P45 (Figure 2B and E), and the staining is compact and localized at P90 (Figure 2C and F). To identify the surface features that may correlate 
with the change in PNNs deposition, we applied contact mode scanning to PNN-positive regions, guided by a fluorescent associate camera (Figure2 G to I). AFM images are $10 \times 10 \mu \mathrm{m}^{2}$ topographical features of the white box delineated in Figure 2 D to F. Topographical measurements on thin sections, originally prepared for histomorphometric analyses, demonstrate a qualitative decrease of roughness of the surface around certain neurons containing PNNs. This change in the topography could be extrapolated to the tissue and may explain the $3 \mathrm{D}$ evolution responsible for the elasticity modification (increase in heterogeneity and $\mathrm{YM}$ ) during maturation.

We next sought to determine topographical features in a surface area of $100^{2} \mu \mathrm{m} 2$ zones and to measure the respective surface roughness. Figure 3 shows representative images obtained for the three cortical areas at the three developmental time points. The left column represents contact mode imaging of PSSC layer II/III, IV and V/VI for P20 (Figure 3A, B and C: blue lines), the center column is P45 (Figure 3E, F and G: black lines) and the right column for P90 (Figure 3I, J, K: purple lines). Intragroup analyses by ANOVA showed no significant difference in roughness, as determined by surface root mean square (Figure3 D, H, L). For example, Rq values of layer II/III, IV and V/VI from P20 were $588 \pm 71,735 \pm 110$ and $743 \pm 103$ respectively (Figure 3D). The measured Rq values for P20 compared to P45 and P90 per $2500 \mu \mathrm{m}^{2}$ were $690 \pm 87,570 \pm 38$ and $256 \pm 10$; indicating a significant intergroup difference in surface roughness (Figure $3 \mathrm{M}, \mathrm{N}$ and $\mathrm{O}$ : blue, black and purple lines) between weaning and juvenile to adult stages. These results are shown at the end of column and row (Figure D, H, L, M, N and O: blue, black and purple lines) which represents the roughness, measured, or calculated for ages and layers, respectively. Two-way ANOVA indicated significant change in Rq with increasing age.

Visual analysis of the AFM images shows the disappearance of small $\left(0-6 \mu \mathrm{m}^{2}\right)$ features over the maturation period, whereas features $>6 \mu \mathrm{m}^{2}$, which represent cell soma, did not change suggesting that the smoothing is not a result of the addition of new cells but rather fine, nanometric structural changes. In an effort to limit observer-dependant bias in the analysis of topographical features of the cortex slices, we implemented a simple image analysis strategy using ImageJ which solely accounted for small features and discarded large feature such as neurons. The visual trend was confirmed by the 
systematic analysis routine, where we found that the number of indentations in the range of $0-5 \mu \mathrm{m}^{2}$, as shown in red in the processed images, consistently decreased with age (Figure 4A, B, C) and is quantified in Figure 3D.

\section{$\underline{\text { Discussion }}$}

Here we show direct measures of tissue elasticity of the different cortical layers of the primary somatosensory area cortical layers II/III, IV and V/VI at different ages from late postnatal to adult, using indentation experiments. To achieve this goal, we used the AFM in the force spectroscopy mode and calculated the Young's modulus of the 9 different conditions, by fitting the indentation curves with the Hertz model (Eq 1). We found an age-dependant shift in the elasticity of the PSSC that correlates with the neuronal plasticity. In fact we obtained similar age -dependant Young's modulus values, ranging from median values of 209 to $790 \mathrm{~Pa}$ for fresh tissues and 400 to $1500 \mathrm{~Pa}$ following a more thorough analyses of the cortical layers of mildly fixed slices, as previously observed (Elkin et al., 2010). We found that even though the coefficient of variation was not significant, the standard deviation increased vastly with age signifying considerable heterogeneity with age. For example, at P90 cortical slices contain elements that range in stiffness from 70PA to 1900PA. Our findings for an increase in the mechanical heterogeneity in the adult cortical layers differ from the previous report on adult cortices (Benjamin S. Elkin et al., 2007; Elkin et al., 2010) ; even if mechanical heterogeneity has been reported for the cortex during embryonic development (Iwashita et al., 2014). We propose that the previously reported absence of heterogeneity in the adult cortex may either be due to the nature of the cortical sampling area, which was the posterior to the frontal areas studied herein; or, the difference may be due the large- $25 \mu \mathrm{m}^{2}$ diameter probe used as compared to the $5 \mu \mathrm{m}^{2}$ in diameter colloidal probe we employed. It is possible that the larger-diameter probe can induce an averaging of the Young's Modulus by increasing the volume of tested material, whereas smaller diameter probes appear to be more sensitive to mechanical evolution of the cortex. Despite the heterogeneity observed in PSSC, the shift toward stiffness in the adult CNS tissues has been largely observed and attributed to the deposition of myelin (Weickenmeier et al., 2016), lipid profile(Li et al., 2017), and, the deposition of the ECM (Elkin et al., 2010). Data obtained in this study are comparable to previously published results of AFM measurements of CNS tissue mechanics, where both rat and mouse brain tissue were 
found to be soft and predominantly elastic (B. S. Elkin et al., 2007; Elkin et al., 2010; Iwashita et al., 2014; Moeendarbary et al., 2017). Further data now suggests that the high spatial resolution of tissue stiffness measured by the AFM may serve to map early, genetic pathophysiological changes in the CNS (Holtzmann et al., 2016). Importantly the authors herein demonstrate the importance for tightly controlling experimental parameters, particularly when measuring living tissue, allowing for the attainment of refined and reproducible data.

While much effort is now placed on understanding the biomechanical properties of CNS tissues, we sought to determine whether we could use topographical nanometric mapping to image and quantify the process of maturation occurring at the PSSC layers. One previous study (Nie et al., 2011) employed the amplitude modulation mode to explored unfixed, cryopreserved rat brain sections to obtain phase images allowing for the first time a topographical image of the cortex. These AFM experiments were conducted in air (40\% humidity) and only on $5^{2} \mu \mathrm{m}^{2}$ or $10^{2} \mu \mathrm{m}^{2}$ areas.The authors demonstrated that AFM phase images were sensitive to subcellular features, which the authors attributed to mechanical contrast, yet nanoscale structural mapping of larger areas was difficult owing to the glycolipid/glycoprotein nature of brain slices. Instead, given that AFM is a high-resolution surface technique applied to a myriad of surfaces (Ludwig et al., 2008), we develop and expand on the biomechanical properties of the PSSC by demonstrating the utility of the AFM contact mode on paraffin embedded tissues to acquire high resolution topographical mapping. We note that the evolution in PSSC stiffness, and thus architecture, correlates with the evolution of the 2D topography of cortical slices. Indeed, understanding the physical topographical organization of the cortex has proven difficult owning to its gel-like consistency. Our findings correspond to the work by (Nie et al., 2011), even if their the measures published were only on $5^{2} \mu \mathrm{m}^{2}$ or $10^{2} \mu \mathrm{m}^{2}$ areas, and expand on these observations as we were able to record contact mode images on $100^{2} \mu \mathrm{m}^{2}$ areas, in liquid environment, and on paraffin embedded slices that allowed for greater sensitivity in the acquisition of topography images. Atomic force microscopy contact mode measures permit for the assessment of surface topography and properties at nanometer scale. The AFM simultaneously produces maps of the surface topography, by generating height images or derived amplitude images, and these permit the 
qualitative and quantitative analyses of surfaces. So far, application of AFM-contact mode is applied technique to collect detailed nanometric information about surface and interface properties respect to other microscopy techniques (Burnham and Colton, 1998; Marrese et al., 2017). Contact mode on fixed, embedded tissues has been applied to biological tissues, where it has been used to identify the fine structure of ocular tissues or skeletal matrix proteins (Antunes et al., 2007; Mass et al., 2014).

Contact mode mapping further permitted the visualization of changes in the topography that coincide with remodelling periods in the PSSC. To date our understanding of cortical function is an integration of the molecular, chemical and electrical behavior in a functional context such as the behaving animal. One often overlooked yet important structure is the ECM. Presently the most widely studied ECM structure in maturation is the lattice like PNNs that enwrap certain interneurons. PNNs are not only representative of maturation, but it is postulated that their spatial arrangement may be indicative of the functional peculiarities of cortical fields (Brückner et al., 1994). Apart from histochemical phenotyping, few markers exist allowing for precise and consistent predictors of maturation. For example, overlapping histological mapping of PNNs with AFM contact mode mapping showed qualitative smoothing of the analysed surface coincides with PNN deposition. Interestingly, we observed a qualitative smoothing of the analysed surface that was confirmed on larger images and quantified using root mean squared roughness measurements of the PNN regions but also of non-PNN containing regions. Since PNNs enwraps only around $30 \%$ of the fast-spiking interneurons, it is likely that the smoothing of the PSSC topography observed during juvenile to adult state are only partially due to PNN deposition.

Notably, it is established that maturation of the juvenile PNN matrix may not be the result of the deposition of PNN components, as many are already present in the immature CNS before PNNs form (Carulli et al., 2007; Galtrey et al., 2008). Our findings indicate that topographical modification may not be the apparition of PNNs but rather a change in the structural organization of these macromolecules that delimit the porosity of the enwrapped neuron and perhaps its surrounding area. Documented changes in topographical features due to organized networks of macromolecules of proteoglycan, especially those containing chitin that are expected to change biomechanical properties, 
are well established in yeast membranes, but not yet shown in other species (Alsteens et al., 2008; Formosa et al., 2013; Schiavone et al., 2014; Touhami et al., 2003). If indeed this is the case in the brain, our results suggest that contact-mode scanning provides important sensitivity to ascertain remodelling of pre-existing macromolecules in the PSSC. Therefore, such an application in the brain may permit for increase in sensitivity and specificity in phenotyping the brain, especially in cases of molecular-phenotypic discordance; for example, PNN content in neurodegenerative or neuropsychiatric disorders(Bitanihirwe and Woo, 2014; Bonneh-Barkay and Wiley, 2009; Morawski et al., 2014).

In this work, we propose an innovative method for measuring changes in the topography of the PSSC sections by use of AFM contact mode topographical mapping. Using this technique, we were able to map nanostructural components in areas up to $100^{2} \mu \mathrm{m}^{2}$, thereby permitting the imaging of the complex architecture indicative of the brain. Moreover, one great advantage of this technique is that paraffin conserved tissues the most commonly used technique in neuroscience and our strategy has the benefit to be applicable towidely used and stored tissues. It is of note that our results are based on developmental periods using an inbred c57BJ strain. In comparing mouse studies it will be important to consider if a similar trajectory is observed, and what genetic or epigenetic modifications can accelerate or perturb this likely process.

New evidence for the biomechanical and viscoelastic properties of specific neuroanatomical regions has enabled alternative means to investigate neural tissues differentially affected in neurodegenerative conditions (Ungureanu et al., 2016), and in trauma (Moeendarbary et al., 2017). Our study shows that application of the AFM force mapping as an innovative means to phenotype the nanomechanical properties of the cortex. In addition, we demonstrate a method to visualize nanoscale characteristics of the lateral PSSC that can be applied to future detailed molecular studies cortical layers. Such ultrastructural changes speculate a more cohesive tissue with increased mechanical stiffness with age. This decrease in surface roughness corresponds not only to critical periods for the ECM deposition but may reflect remodeling factors that regulate structural and functional aspects of circuit development including, dendritic arborization and synaptogenesis, or others that have yet to be 
identified. An important finding of our work is that the FC remains moderately porous irrespective of layer and cortical sub-region. These results may suggest that porosity enables input and circuitry remodeling to enable continued plasticity. Taken together our results suggest that application of the AFM to study cyto-architechture and biomechanics of brain tissues may help identify morphometric markers in neurodevelopmental and neurodegenerative diseases that precede the onset of psychosocial phenotypes. 
Conflict of Interest: The authors declare that they have no conflict of interest.

Acknowledgements. We thank Randall Willis and Laure Verret for critically reading the manuscript. We thank Cedric Baudelin and Lionel Mouledous for their technical expertise. We thank Lucie Fontaine at the Histology Platform, and, Yvan Nicaise and Mélanie Pucelle at the Imaging Platform (CMEAB). This work was supported by the "Fondation pour la Recherche Médicale" (G.S.; grant ING21040129094) and by the "les initiatives d'excellence" at the University de Toulouse (IDEX, S11V9R49). ED and DA are researchers at the Centre National de la Recherche Scientifique (CNRS). 
References

Alsteens, D., Dupres, V., Evoy, K.M., Wildling, L., Gruber, H.J., Dufrêne, Y.F., 2008. Structure, cell wall elasticity and polysaccharide properties of living yeast cells, as probed by AFM. Nanotechnology 19, 384005. doi:10.1088/0957-4484/19/38/384005

Antunes, A., Gozzo, F.V., Borella, M.I., Nakamura, M., Safatle, M.V., Barros, P.S.M., Toma, H.E., 2007. Atomic Force Imaging of Ocular Tissues: morphological study of healthy and cataract lenses, in: Modern Research and Educational Topics in Microscopy. Mendez-Villas et Diaz, Spain, Badajoz, pp. 29-36.

Beaussart, A., El-Kirat-Chatel, S., Sullan, R.M.A., Alsteens, D., Herman, P., Derclaye, S., Dufrêne, Y.F., 2014. Quantifying the forces guiding microbial cell adhesion using single-cell force spectroscopy. Nat. Protoc. 9, 1049-1055. doi:10.1038/nprot.2014.066

Bernard, C., Prochiantz, A., 2016. Otx2-PNN Interaction to Regulate Cortical Plasticity. Neural Plast. 2016, 7931693. doi:10.1155/2016/7931693

Bitanihirwe, B.K.Y., Woo, T.-U.W., 2014. Perineuronal nets and schizophrenia: the importance of neuronal coatings. Neurosci. Biobehav. Rev. 45, 85-99. doi:10.1016/j.neubiorev.2014.03.018

Bonneh-Barkay, D., Wiley, C.A., 2009. Brain extracellular matrix in neurodegeneration. Brain Pathol. Zurich Switz. 19, 573-585. doi:10.1111/j.1750-3639.2008.00195.x

Brückner, G., Seeger, G., Brauer, K., Härtig, W., Kacza, J., Bigl, V., 1994. Cortical areas are revealed by distribution patterns of proteoglycan components and parvalbumin in the Mongolian gerbil and rat. Brain Res. 658, 67-86. doi:10.1016/S0006-8993(09)90012-9

Burnham, N.A., Colton, R.J., 1998. Measuring the nanomechanical properties and surface forces of materials using an atomic force microscope. J. Vac. Sci. Technol. Vac. Surf. Films. doi:10.1116/1.576168

Callaghan, B.L., Graham, B.M., Li, S., Richardson, R., 2013. From resilience to vulnerability: mechanistic insights into the effects of stress on transitions in critical period plasticity. Front. Psychiatry 4, 90. doi:10.3389/fpsyt.2013.00090

Carstens, K.E., Phillips, M.L., Pozzo-Miller, L., Weinberg, R.J., Dudek, S.M., 2016. Perineuronal Nets Suppress Plasticity of Excitatory Synapses on CA2 Pyramidal Neurons. J. Neurosci. Off. J. Soc. Neurosci. 36, 6312-6320. doi:10.1523/JNEUROSCI.0245-16.2016

Croteau-Chonka, E.C., Dean III, D.C., Remer, J., Dirks, H., O'Muircheartaigh, J., Deoni, S.C.L., 2016. Examining the relationships between cortical maturation and white matter myelination throughout early childhood. Neurolmage 125, 413-421. doi:10.1016/j.neuroimage.2015.10.038

Di Cristo, G., Chattopadhyaya, B., Kuhlman, S.J., Fu, Y., Bélanger, M.-C., Wu, C.Z., Rutishauser, U., Maffei, L., Huang, Z.J., 2007. Activity-dependent PSA expression regulates inhibitory maturation and onset of critical period plasticity. Nat. Neurosci. 10, 1569-1577. doi:10.1038/nn2008

Elkin, B.S., Azeloglu, E.U., Costa, K.D., Morrison, B., 2007. Mechanical heterogeneity of the rat hippocampus measured by atomic force microscope indentation. J. Neurotrauma 24,812 822. doi:10.1089/neu.2006.0169

Elkin, B.S., Azeloglu, E.U., Costa, K.D., Morrison, B., 2007. Mechanical heterogeneity of the rat hippocampus measured by atomic force microscope indentation. J Neurotrauma 24, 812-22. doi:10.1089/neu.2006.0169

Elkin, B.S., llankovan, A., Morrison, B., 2010. Age-dependent regional mechanical properties of the rat hippocampus and cortex. J. Biomech. Eng. 132, 011010. doi:10.1115/1.4000164

Emerson, R.J., Camesano, T.A., 2006. On the importance of precise calibration techniques for an atomic force microscope. Ultramicroscopy 106, 413-422. doi:10.1016/j.ultramic.2005.11.008

Formosa, C., Schiavone, M., Martin-Yken, H., François, J.M., Duval, R.E., Dague, E., 2013. Nanoscale Effects of Caspofungin against Two Yeast Species, Saccharomyces cerevisiae and Candida albicans. Antimicrob. Agents Chemother. 57, 3498-3506. doi:10.1128/AAC.00105-13 
Fox, K., Wong, R.O.L., 2005. A Comparison of Experience-Dependent Plasticity in the Visual and Somatosensory Systems. Neuron 48, 465-477. doi:10.1016/j.neuron.2005.10.013

Franze, K., Janmey, P.A., Guck, J., 2013. Mechanics in neuronal development and repair. Annu. Rev. Biomed. Eng. 15, 227-251. doi:10.1146/annurev-bioeng-071811-150045

Giedd, J.N., Blumenthal, J., Jeffries, N.O., Castellanos, F.X., Liu, H., Zijdenbos, A., Paus, T., Evans, A.C., Rapoport, J.L., 1999. Brain development during childhood and adolescence: a longitudinal MRI study. Nat. Neurosci. 2, 861-863. doi:10.1038/13158

Hensch, T.K., 2005. Critical period plasticity in local cortical circuits. Nat Rev Neurosci 6, 877-88. doi:10.1038/nrn1787

Holtzmann, K., Gautier, H.O.B., Christ, A.F., Guck, J., K?rad?ttir, R.T., Franze, K., 2016. Brain tissue stiffness is a sensitive marker for acidosis. J. Neurosci. Methods 271, 50-54. doi:10.1016/j.jneumeth.2016.07.002

Iwashita, M., Kataoka, N., Toida, K., Kosodo, Y., 2014. Systematic profiling of spatiotemporal tissue and cellular stiffness in the developing brain. Dev. Camb. Engl. 141, 3793-3798. doi:10.1242/dev.109637

J. Roa, J., Oncins, G., Diaz, J., Sanz, F., Segarra, M., 2011. Calculation of Young's Modulus Value by Means of AFM. Recent Pat. Nanotechnol. 5, 27-36. doi:10.2174/187221011794474985

Johnson, C.M., Loucks, F.A., Peckler, H., Thomas, A.W., Janak, P.H., Wilbrecht, L., 2016a. Long-range orbitofrontal and amygdala axons show divergent patterns of maturation in the frontal cortex across adolescence. Dev. Cogn. Neurosci. 18, 113-120. doi:10.1016/j.den.2016.01.005

Johnson, C.M., Peckler, H., Tai, L.-H., Wilbrecht, L., 2016b. Rule learning enhances structural plasticity of long-range axons in frontal cortex. Nat. Commun. 7, 10785. doi:10.1038/ncomms10785

Li, Q., Bozek, K., Xu, C., Guo, Y., Sun, J., Pääbo, S., Sherwood, C.C., Hof, P.R., Ely, J.J., Li, Y., Willmitzer, L., Giavalisco, P., Khaitovich, P., 2017. Changes in Lipidome Composition during Brain Development in Humans, Chimpanzees, and Macaque Monkeys. Mol. Biol. Evol. 34, 1155. doi:10.1093/molbev/msx065

Litinetsky, L., Barkay, Z., Kalicharan, D., Rosenzweig, E., Ishay, J.S., 2002. AFM study of microstructures on the cornea of the compound eye and ocelli of the hornet Vespa orientalis (Insecta, Hymenoptera). Physiol. Chem. Phys. Med. NMR 34, 61-69.

Ludwig, T., Kirmse, R., Poole, K., Schwarz, U.S., 2008. Probing cellular microenvironments and tissue remodeling by atomic force microscopy. Pflüg. Arch. - Eur. J. Physiol. 456, 29-49. doi:10.1007/s00424-007-0398-9

MacGregor, D.G., Chesler, M., Rice, M.E., 2001. HEPES prevents edema in rat brain slices. Neurosci Lett 303, 141-4.

Marrese, M., Guarino, V., Ambrosio, L., 2017. Atomic Force Microscopy: A Powerful Tool to Address Scaffold Design in Tissue Engineering. J. Funct. Biomater. 8. doi:10.3390/jfb8010007

Marsh, R., Gerber, A.J., Peterson, B.S., 2008. Neuroimaging Studies of Normal Brain Development and Their Relevance for Understanding Childhood Neuropsychiatric Disorders. J. Am. Acad. Child Adolesc. Psychiatry 47, 1233-1251. doi:10.1097/CHI.0b013e318185e703

Mass, T., Drake, J.L., Peters, E.C., Jiang, W., Falkowski, P.G., 2014. Immunolocalization of skeletal matrix proteins in tissue and mineral of the coral Stylophora pistillata. Proc. Natl. Acad. Sci. 111, 12728-12733. doi:10.1073/pnas.1408621111

Moeendarbary, E., Weber, I.P., Sheridan, G.K., Koser, D.E., Soleman, S., Haenzi, B., Bradbury, E.J., Fawcett, J., Franze, K., 2017. The soft mechanical signature of glial scars in the central nervous system. Nat. Commun. 8. doi:10.1038/ncomms14787

Morawski, M., Filippov, M., Tzinia, A., Tsilibary, E., Vargova, L., 2014. ECM in brain aging and dementia. Prog. Brain Res. 214, 207-227. doi:10.1016/B978-0-444-63486-3.00010-4

Nie, H.-Y., Taylor, A.R., Lau, W.M., MacFabe, D.F., 2011. Subcellular features revealed on unfixed rat brain sections by phase imaging. The Analyst 136, 2270-2276. doi:10.1039/c1an15125h

Nieto-Gonzalez, J.L., Jensen, K., 2013. BDNF Depresses Excitability of Parvalbumin-Positive Interneurons through an M-Like Current in Rat Dentate Gyrus. PLOS ONE 8, e67318. doi:10.1371/journal.pone.0067318 
Paus, T., Keshavan, M., Giedd, J.N., 2008. Why do many psychiatric disorders emerge during adolescence? Nat. Rev. Neurosci. 9, 947-957. doi:10.1038/nrn2513

Schiavone, M., Vax, A., Formosa, C., Martin-Yken, H., Dague, E., François, J.M., 2014. A combined chemical and enzymatic method to determine quantitatively the polysaccharide components in the cell wall of yeasts. FEMS Yeast Res. 14, 933-947. doi:10.1111/1567-1364.12182

Semple, B.D., Blomgren, K., Gimlin, K., Ferriero, D.M., Noble-Haeusslein, L.J., 2013. Brain development in rodents and humans: Identifying benchmarks of maturation and vulnerability to injury across species. Prog. Neurobiol. 0, 1-16. doi:10.1016/j.pneurobio.2013.04.001

Somerville, L.H., 2016. Searching for Signatures of Brain Maturity: What Are We Searching For? Neuron 92, 1164-1167. doi:10.1016/j.neuron.2016.10.059

Spedden, E., Staii, C., 2013. Neuron biomechanics probed by atomic force microscopy. Int. J. Mol. Sci. 14, 16124-16140. doi:10.3390/ijms140816124

Takesian, A.E., Hensch, T.K., 2013. Balancing plasticity/stability across brain development. Prog. Brain Res. 207, 3-34. doi:10.1016/B978-0-444-63327-9.00001-1

Touhami, A., Nysten, B., Dufrêne, Y.F., 2003. Nanoscale Mapping of the Elasticity of Microbial Cells by Atomic Force Microscopy. Langmuir 19, 4539-4543. doi:10.1021/la034136x

Ungureanu, A.-A., Benilova, I., Krylychkina, O., Braeken, D., De Strooper, B., Van Haesendonck, C., Dotti, C.G., Bartic, C., 2016. Amyloid beta oligomers induce neuronal elasticity changes in age-dependent manner: a force spectroscopy study on living hippocampal neurons. Sci. Rep. 6, 25841. doi:10.1038/srep25841

Weickenmeier, J., de Rooij, R., Budday, S., Steinmann, P., Ovaert, T.C., Kuhl, E., 2016. Brain stiffness increases with myelin content. Acta Biomater 42, 265-72. doi:10.1016/j.actbio.2016.07.040

Zhang, P., Yang, Y., Candiello, J., Thorn, T.L., Gray, N., Halfter, W.M., Hu, H., 2013. Biochemical and biophysical changes underlie the mechanisms of basement membrane disruptions in a mouse model of dystroglycanopathy. Matrix Biol. J. Int. Soc. Matrix Biol. 32, 196-207. doi:10.1016/j.matbio.2013.02.002 
Figure 1. Increase in PSSC stiffness and heterogeneity during maturation

(A) Schematic representation of the methodology employed. (B) Force curves measured on 400uMthick fresh cortical slices obtained from subjects aged p20, p45 and p90. An AFM colloidal probe was used and measurements were obtained by 40 dispersed points throughout the entire cortical area. (C) Force curves measured in the 3 cortical layers of p20, p45 and p90 400uM-thick sections of frontal cortices. An AFM colloidal probe was used and measurements were obtained by 35-40 dispersed points throughout $100^{2} \mu \mathrm{m}^{2}$ area within layer II/III, IV and V/VI. Young's modulus of each region and each cohort was calculated from the obtained force-distance curves. While the Young modulus was not different for cortical layers within each age cohort, an increase in heterogeneity and stiffness is observed between the age groups as determined by 2 -way ANOVA. $* \mathrm{p}<0.5, * * \mathrm{p}<0.01$.

Figure 2. Histochemical and topographical mapping of PSSC maturation

Representative immunofluorescence images show PNNs (WFA staining, red) and DAPI as a nuclear counterstain on p20(A) p45 (B) and p90 (C) PSSCs. D-F are high magnifications of the inlets of WFA stained areas. Characteristic age-related increase in PNNs, as diffusely deposited at p45, and concentrated assemblies were observed at p90. G-I Topographical mapping of inlet areas $\left(10 \times 10 \mu \mathrm{m}^{2}\right)$ defined in D-F of areas surrounding neuronal silhouettes using the scanning mode on the AFM. A condensation and smoothing of the area is observed that coincides with the deposition of the PNN.

Figure 3. Topographical mapping and $R q$ measurements of PSSC maturation

Atomic force microscopy scanning mode image show representative height images obtained for the three cortical areas at the three developmental time points. Height images were obtained in contact mode by using the following scanning conditions: force set point $5 \mathrm{nN}$, scanning frequency $1 \mathrm{~Hz}$, images resolution $512^{2}$ pixels on a scan area of $100^{2} \mu \mathrm{m}^{2}$. The zero point of the bar corresponds to the lowest measured point in the image; the upper limit of the bar is the highest point. The level bar is adjusted to obtain the best contrast for the picture. The left column represents contact mode imaging for P20 (A, B and C; color coded with blue lines), the middle for p45 (E, F and G; color coded with black lines) and the right column for p90 (I, J, and K; color coded with purple lines). The top row represents layer II/III (A, E and I; blue, black and purple dotted lines respectively), the middle row is layer IV (Figure B, F, and J; blue, black and purple dotted lines respectively), and the bottom row is layer V and VI (C, J, and K; blue, black and purple dotted lines respectively). Graphs representing quantified roughness for each cortical layer when comparing the 3 different age groups; $n=3$ /group row (D, H, L, M, N and $\mathrm{O}$ ). ${ }^{*} \mathrm{P}<0.5$, ** $\mathrm{P}<0.01$ using 2-way ANOVA.

Figure 4. Particle size quantification as a measure of the PSSC smoothing phenotype

ImageJ-processed AFM scanning mode images of a p20 (A) p45 (B) and p90 (C) PSSC slices and automated feature detection. The backgrounds are grey scale topography micrographs, and the features automatically detected are shown in red. D: plot of number of features detected as a function of their area for P20, P45 and P90 are represented in red. Features larger than $6 \mu \mathrm{m}^{2}$ were automatically excluded from the analysis. 